\title{
Wilson-Polyakov loop at finite temperature in large- $N$ gauge theory and anti-de Sitter supergravity *
}

\author{
Soo-Jong Rey ${ }^{\mathrm{a}}$, Stefan Theisen ${ }^{\mathrm{b}}$, Jung-Tay Yee ${ }^{\mathrm{a}}$ \\ a Physics Department, Seoul National University, Seoul 151-742, South Korea \\ b Sektion Physik, Universität München, D-80333 München, Germany
}

Received 31 March 1998; accepted 17 June 1998

\begin{abstract}
Aspects of $d=4, \mathcal{N}=4$ superconformal $U(N)$ gauge theory are studied at finite temperature. Utilizing dual description of large- $N$ and strong coupling limit via Type IIB string theory compactification on Schwarzschild-anti-de Sitter space-time, we study correlations of Wilson-Polyakov loops and heavy quark potential thereof. We find that the heavy quark potential is Coulomb-like and has a finite range, as expected for gauge theory in high temperature, deconfinement phase. The potential exhibits finite temperature scaling consistent with underlying conformal invariance. We also study isolated heavy quark on a probe D3-brane world-volume and find supporting evidence that near extremal D3-branes are located at the Schwarzschild horizon. (C) 1998 Elsevier Science B.V.
\end{abstract}

PACS: 11.25.-w; 11.10.Wx; 11.15.-q

Keywords: QCD; Supergravity; Non-perturbative effects; Finite temperature; Wilson loop

\section{Introduction}

To understand the large- $N$ and classical strong coupling behavior of $S U(N)$ gauge theories has been an open problem for decades [1,2]. Recently, with better understanding of D-brane world-volume dynamics, connections to string theory and therefore new

\footnotetext{
* Work supported in part by KOSEF SRC-Program, Ministry of Education Grant BSRI 97-2418, SNU Faculty Research Fund and Korea Foundation for Advanced Studies Faculty Fellowship, by GIF - the German-Israeli Foundation for Scientific Research and by the European Commission TMP Programme ERBFMRX-CT960045 .
} 
approaches to the problem have become available. Built on earlier studies of the nearhorizon geometry of $\mathrm{D}$ - and $\mathrm{M}$-branes and their absorption and Hawking emission processes [3], Maldacena has put forward a proposal [4] for the large- $N$ limit of world-volume quantum field theories. In particular, the gauge theory Green functions can be calculated via $S$-matrix elements of anti-de Sitter supergravity [5,6].

Among field theories studied so far, the most tractable case is $d=4, \mathcal{N}=4$ supersymmetric gauge theory with gauge group $S U(N)$ (and truncations to $\mathcal{N}=2,1,0$ theories with vanishing beta functions) [7]. The theory is superconformally invariant and is realized as the world-volume theory of $N$ coincident D3-branes in Type IIB string theory. The latter configuration induces the near-horizon geometry of ad $S_{5} \times S_{5}$

$$
d s_{\text {extreme }}^{2}=\alpha^{\prime}\left[\frac{\mathrm{U}^{2}}{g_{\text {eff }}}\left(-d t^{2}+d \mathbf{x}_{\|}^{2}\right)+\frac{g_{\text {eff }}}{\mathrm{U}^{2}} d \mathrm{U}^{2}+g_{\text {eff }} d \Omega_{\mathbf{5}}^{2}\right]
$$

whose radius of curvature-squared is $g_{\mathrm{eff}}=g_{\mathrm{YM}}^{2} N$, where $g_{\mathrm{YM}}^{2}=\lambda_{\text {IIB }}$, and whose FreundRubin background is provided by the self-dual flux $Q_{5}=\oint_{S_{5}} H_{5}=\oint_{S_{5}}{ }^{*} H_{5}=N$. Hence, in the large- $N$ and strong coupling $g_{\text {eff }} \gg 1$ limit, the gauge theory is dual to Type IIB supergravity on $a d S_{5} \times S_{5}$.

Maldacena's proposal is not restricted to zero temperature and can be extended to the study of thermodynamics at finite temperature. At finite temperature, the large- $N$ and strong coupling limit of $d=4, \mathcal{N}=4$ supersymmetric gauge theory is dual to the near-horizon geometry of near extremal D3-branes [8] in Type IIB string theory. The latter is given by a Schwarzschild-anti-de Sitter Type IIB supergravity compactification:

$$
d s^{2}=\alpha^{\prime}\left[\frac{1}{\sqrt{G}}\left(-H d t^{2}+d \mathbf{x}_{\|}^{2}\right)+\sqrt{G}\left(\frac{1}{H} d \mathrm{U}^{2}+\mathrm{U}^{2} d \Omega_{5}^{2}\right)\right],
$$

where

$$
\begin{aligned}
& G \equiv \frac{g_{\text {eff }}^{2}}{\mathrm{U}^{4}}, \\
& H \equiv 1-\frac{\mathrm{U}_{0}^{4}}{\mathrm{U}^{4}} \quad\left(\mathrm{U}_{0}^{4}=\frac{2^{7} \pi^{4}}{3} g_{\text {eff }}^{4} \frac{\mu}{N^{2}}\right) .
\end{aligned}
$$

The parameter $\mu$ is interpreted as the free energy density on the near extremal D3brane, hence, $\mu=\left(4 \pi^{2} / 45\right) N^{2} T^{4}$. In the field theory limit $\alpha^{\prime} \rightarrow 0, \mu$ remains finite. In turn, the proper energy $E_{\text {sugra }}=\sqrt{g_{\text {eff }} / \alpha^{\prime}} \mu / \mathrm{U}$ and the dual description in terms of modes propagating in the above supergravity background is expected to be a good approximation.

At a finite critical temperature $T=T_{c}$, pure $S U(N)$ gauge theory exhibits a deconfinement phase transition $[9,10]$. The relevant order parameter is the Wilson-Polyakov loop:

$$
P(\mathbf{x})=\frac{1}{N} \operatorname{Tr} \mathcal{P} \exp \left(i \int_{0}^{1 / T} A_{0}(\mathbf{x}) d t\right)
$$


Below the critical temperature $T<T_{c},\langle P\rangle=0$ and QCD confines. Above $T>T_{c},\langle P\rangle$ is non-zero and takes values in $\mathbb{Z}_{N}$, the center group of $S U(N) .{ }^{1}$ Likewise, the two-point correlation of parallel Wilson-Polyakov loops

$$
\Gamma(\mathbf{d}, T) \equiv\left\langle P^{\dagger}(\mathbf{0}) P(\mathbf{d})\right\rangle_{T}=e^{-\mathcal{F}(\mathbf{d}, T) / T} \approx e^{-V_{\overline{\mathrm{Q}}}(\mathbf{d}, T) / T}
$$

measures the static potential at finite temperature between quark and anti-quark separated by a distance $d$.

At sufficiently high temperature, thermal excitations produce a plasma of quarks and gluons and gives rise to Debye mass $m_{\mathrm{E}} \approx g_{\mathrm{eff}} T$ (which is responsible for screening the color electric flux) and magnetic mass $m_{\mathrm{M}} \approx g_{\mathrm{eff}}^{2} T$ (which corresponds to the glueball mass gap in the confining three-dimensional pure gauge theory). Their effects are captured by the asymptotic behavior of the heavy quark potential [13]:

$$
\begin{aligned}
V_{\mathrm{QQ}}(\mathbf{d}, T) \approx & -C_{\mathrm{E}} \frac{1}{|\mathbf{d}|^{2}} e^{-2 m_{\mathrm{E}}|\mathbf{d}|}+\ldots, & C_{\mathrm{E}}=\mathcal{O}\left(g_{\mathrm{YM}}^{4}\right) \\
& -C_{\mathrm{M}} \frac{1}{|\mathbf{d}|} e^{-m_{\mathrm{M}}|\mathbf{d}|}+\ldots, & C_{\mathrm{M}}=\mathcal{O}\left(g_{\mathrm{YM}}^{12}\right) .
\end{aligned}
$$

In this paper, utilizing the aforementioned correspondence, we study correlators of Wilson-Polyakov loops and heavy quark potential at finite temperature in $d=4, \mathcal{N}=4$ supersymmetric gauge theory. At zero temperature, heavy quark potential has been studied recently [14,15] and Coulomb-type behavior consistent with the underlying conformal invariance has been observed. ${ }^{2}$ We will find that, at finite temperature, the heavy quark potential exhibits short-range asymptotic behavior as in Eq. (6), but again in a manner fully respecting the underlying conformal invariance. We will also study an isolated single quark excitation on a probe D3-brane and find an indication that the near extremal D3-branes cannot reside behind the Schwarzschild horizon. This provides further support to the Maldacena's earlier observation [18] that near-extremal D3-branes are located at the horizon.

Concurrent with the first version of this paper, a preprint by Witten [19] and a preprint by Brandhuber, Itzhaki, Sonnenschein and Yankielowicz [20] have appeared. The general results of Witten on the impossibility of having a phase-transition at finite temperature for the $N=4$ theory led us to reconsider related speculations in the original version of our paper. This also eliminated discrepancies in interpretation with the second paper mentioned.

\section{Static quark potential at finite temperature}

We want to study the dynamics of a test Type IIB string which ends on a nearextremal D3-brane. Denote the string coordinates by $X^{\mu}(\sigma, \tau)$, where $\sigma, \tau$ parametrize

\footnotetext{
${ }^{1}$ For a review of gauge theory at finite temperature, see, for example, Refs. $[11,12]$.

${ }^{2}$ Quark-monopole potential has also been examined $|16|$ utilizing a triple string junction |17].
} 
the string world-sheet. Low-energy dynamics of the test string may be described via its Nambu-Goto action, whose Lagrangian is ${ }^{3}$

$$
L_{\mathrm{NG}}=-\int d \sigma \sqrt{-\operatorname{det} h_{a b}}+L_{\text {boundary }}
$$

Here, the induced metric on the world-sheet is

$$
h_{a b}=G_{\mu \nu}(X) \partial_{a} X^{\mu} \partial_{b} X^{\nu} .
$$

To study the relevant string configurations of our interest, we take $X^{0}=t=\tau$ and decompose the nine spatial embedding coordinates into components parallel and perpendicular to the D3-branes:

$$
\mathbf{X}=\left(\mathbf{X}_{\|}, \alpha^{\prime} \mathrm{U}, \alpha^{\prime} \mathrm{U} \Omega_{5}\right)
$$

In the background metric, Eq. (2), straightforward calculation yields $\left(\equiv \partial_{t},{ }^{\prime} \equiv \partial_{\sigma}\right)$

$$
\begin{aligned}
& h_{00}=\frac{1}{\sqrt{G}}\left(-H+\dot{\mathbf{X}}_{\|}^{2}\right)+\sqrt{G}\left(\frac{1}{H} \dot{\mathrm{U}}^{2}+\mathrm{U}^{2} \dot{\Omega}_{5}^{2}\right), \\
& h_{11}=\frac{1}{\sqrt{G}} \mathbf{X}_{\|}^{\prime 2}+\sqrt{G}\left(\frac{1}{H} \mathrm{U}^{\prime 2}+\mathrm{U}^{2} \Omega_{5}^{\prime 2}\right), \\
& h_{01}=\frac{1}{\sqrt{G}} \dot{\mathbf{X}}_{\|} \cdot \mathbf{X}_{\|}^{\prime}+\sqrt{G}\left(\frac{1}{H} \dot{\mathrm{U}} \mathrm{U}^{\prime}+\mathrm{U}^{2} \dot{\Omega}_{5} \cdot \Omega_{5}^{\prime}\right) .
\end{aligned}
$$

From this, it is easy to see that a static configuration is described by

$$
L_{\mathrm{NG}} \rightarrow-\int d \sigma \sqrt{\mathrm{U}^{\prime 2}+H \mathrm{U}^{2} \Omega_{5}^{\prime 2}+\frac{H}{G} \mathbf{X}_{\|}^{\prime 2}} .
$$

Recalling that $G$ and $H$ are functions of $\left|\mathbf{X}_{\perp}\right|=\alpha^{\prime} U$ only, the equations of motion are

$$
\begin{aligned}
& \left(\frac{\frac{H}{G} \mathbf{X}_{\|}^{\prime}}{\sqrt{\mathrm{U}^{\prime 2}+H \mathrm{U}^{2} \Omega_{5}^{\prime 2}+\frac{H}{G} \mathbf{X}_{\|}^{\prime 2}}}\right)^{\prime}=0 \\
& \left(\frac{H \mathrm{U}^{2} \Omega_{5}^{\prime}}{\sqrt{\mathrm{U}^{\prime 2}+H U^{2} \Omega_{5}^{\prime 2}+\frac{H}{G} \mathbf{X}_{\|}^{\prime 2}}}\right)^{\prime}=0 \\
& \left(\frac{\mathrm{U}^{\prime}}{\sqrt{\mathrm{U}^{\prime 2}+H \mathrm{U}^{2} \Omega_{5}^{\prime 2}+\frac{H}{G} \mathbf{X}_{\|}^{\prime 2}}}\right)^{\prime}=\frac{1}{2} \frac{\mathbf{X}_{\|}^{\prime 2} \partial_{\mathrm{U}}\left(\frac{H}{G}\right)+\Omega_{5}^{\prime 2} \partial_{\mathrm{U}}\left(H \mathrm{U}^{2}\right)}{\sqrt{\mathrm{U}^{\prime 2}+H \mathrm{U}^{2} \Omega_{5}^{\prime 2}+\frac{H}{G} \mathbf{X}_{\|}^{\prime 2}}} .
\end{aligned}
$$

Following the prescription of Refs. [14,15], one can now look for possible static string configurations that represent a pair of heavy quark and anti-quark. These strings are infinitely stretched out of the $a d S_{5}$ boundary and act as static color sources of the boundary superconformal Yang-Mills theory. As in the zero temperature situation [14,15], we will

\footnotetext{
${ }^{3}$ Throughout the paper, unless explicitly specified, we suppress the string tension $1 / 4 \pi \alpha^{\prime}=1$.
} 


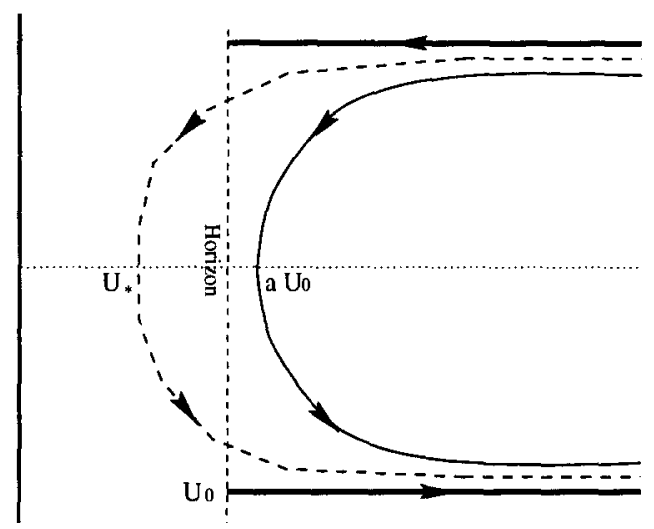

Fig. 1. Possible macroscopic string configurations representing various timelike Polyakov-Wilson loops at finite temperature. The Schwarzschild horizon is located at $\alpha^{\prime} \mathrm{U}_{0}$. The first is a pair of straight, oppositely oriented strings stretched between the $a d S_{5}$ boundary and the Schwarzschild horizon. The second is a $\mathrm{U}$-shaped string running between quark and anti-quark. The string tip is the closest point to the horizon and is located at $a \mathrm{U}_{0}$. For comparison, the $\mathrm{U}$-shape string at zero temperature is also shown.

find that there are two possible string geodesics. The first is a pair of oppositely oriented straight macroscopic strings. At finite temperature, a novelty of this configuration is that both strings end at $\alpha^{\prime} \mathrm{U}_{0}$, and not at $\alpha^{\prime} \mathrm{U}=0$. This is a consequence of Maldacena's observation [18] that near extremal $N$ D3-branes are located at the Schwarzschild horizon. The second is a U-shaped string inter-connecting quark and anti-quark. The two possible configurations are illustrated in Fig. 1.

We begin with the first configuration: a pair of straight macroscopic strings. The strings are stretched between $\alpha^{\prime} U=\infty$, the boundary of $a d S_{5}$, and the Schwarzschild horizon, $\alpha^{\prime} \mathrm{U}_{0}$ :

$$
\mathrm{U}=\sigma, \quad \mathrm{X}_{\|}=\text {constant }, \quad \Omega_{5}=\text { constant. }
$$

Each macroscopic string of opposite orientation is a realization of timelike WilsonPolyakov loops $\langle P\rangle,\left\langle P^{\dagger}\right\rangle$ in Eq. (4). Likewise, two parallel macroscopic strings of opposite orientation (parametrized as in Eq. (13) ) at a separation $|\mathbf{d}|$ is a realization of the two-point Wilson-Polyakov loop correlator $\left\langle P^{\dagger}(0) P(d)\right\rangle_{T}$ in Eq. (5). As the two are oriented oppositely but have the same $S O(6)$ angular position $\Omega_{5}$, the supersymmetry is completely broken and an attractive force will act between them. The force has no visible effect, however, as the quarks are heavy enough. In Fig. 1, we have illustrated the configuration running along the $\alpha^{\prime} U$-direction. Regularizing the string length by $A=\mathrm{U} / \mathrm{U}_{0}$, the total energy of the quark pair $E_{\mathrm{Q}}$ (measured in gauge theory unit) turns out equal to the mass of the quark pair at temperature $T$ :

$$
E_{\mathrm{QQ}}(T, d)=2(\Lambda-1) \mathrm{U}_{0}=2 M_{\mathrm{Q}} ; \quad(\Lambda \rightarrow \infty) .
$$

Of some interest is the fluctuation dynamics of the macroscopic strings. The dynamics would be relevant for understanding Hawking radiation from the Schwarzschild-anti-de Sitter black hole (2) along the string and the thermal distribution of string configura- 
tions. From Eqs. (7), (10), one finds that harmonic fluctuations are governed by the Lagrangian

$$
L^{(2)}=\frac{1}{2}\left[\left(\frac{1}{H} \dot{\mathbf{X}}_{\|}^{2}-\frac{H}{G} \mathbf{X}_{\|}^{\prime 2}\right)+\left(\frac{G}{H} \mathrm{U}^{2} \dot{\Omega}_{5}^{2}-H \mathrm{U}^{2}{\Omega_{5}^{\prime}}^{2}\right)\right]+\ldots
$$

Introducing Schwarzschild-anti-de Sitter tortoise coordinate,

$$
r=\int d \mathrm{U} \frac{\sqrt{G}}{H},
$$

one finds that the equations of motion may be expressed as

$$
\left[\partial_{t}^{2}-\partial_{r}^{2}+M^{2}(r)\right] \mathbf{Y}_{\|}(r, t)=0
$$

where $\mathbf{Y}_{\|}(r, t)=\mathrm{U} \mathbf{X}_{\|}$and

$$
M^{2}(r)=\frac{2}{g_{\mathrm{eff}}^{2}} \mathrm{U}^{2}\left(1-\frac{\mathrm{U}_{0}^{8}}{\mathrm{U}^{8}}\right)
$$

and

$$
\left[\partial_{t}^{2}-\partial_{r}^{2}\right] \Omega_{5}(r, t)=0 .
$$

Fluctuations of the transverse field $\mathbf{Y}_{\|}$obey the Klein-Gordon equation with spatially varying mass $M(r)$. Outside the Schwarzschild horizon, $M(r)$ is always positive and grows indefinitely as the boundary of $a d S_{5}$ is approached. As this renders normalizable solutions, harmonic fluctuations along the $\mathbf{X}_{\|}$direction remain always bounded and stable. Fluctuations of the angular field $\Omega_{5}$ are free, hence, form a $(1+1)$-dimensional Bose gas at Hawking temperature $T_{H}=\mathrm{U}_{0} / \pi g_{\text {eff }}$. Logarithmic infrared divergence of the latter system will then cause the classical string configuration to wander on $S_{5}$. Over a time interval $\Delta \tau$, for example, the thermal ensemble average yields

$$
\left\langle\Omega_{5}^{2}\right\rangle_{T} \approx 5 \frac{T_{H}}{2 \pi} \cdot \Delta \tau
$$

At large- $N$ and strong coupling $g_{\text {eff }} \rightarrow \infty, M(r)$ vanishes and $\mathbf{Y}_{\|}$fields exhibit thermal infrared divergence as well. In turn, fluctuation of the $\mathbf{X}_{\|}$field is estimated

$$
\left\langle\mathbf{X}_{\|}^{2}\right\rangle_{T} \approx 3 \frac{1}{\mathrm{U}^{2}} \frac{T_{H}}{2 \pi} \cdot \Delta \tau
$$

The fluctuation is most pronounced near the Schwarzschild horizon.

We now turn to the second configuration: a macroscopic U-shape string whose each end is connected to quark and anti-quark at the boundary of ad $S_{5}$. Parametrizing the string via static gauge:

$$
\mathbf{X}_{\|}=\sigma \hat{\mathbf{n}}, \quad \Omega_{5}=\text { constant },
$$

the equations of motion 


$$
\begin{aligned}
& \left(\frac{\frac{H}{G}}{\sqrt{\mathrm{U}^{2}+\frac{H}{G}}}\right)^{\prime}=0, \\
& \left(\frac{\mathrm{U}^{\prime}}{\sqrt{\mathrm{U}^{\prime 2}+\frac{H}{G}}}\right)^{\prime}=\frac{\partial \mathrm{U}\left(\frac{H}{G}\right)}{\sqrt{\mathrm{U}^{2}+\frac{H}{G}}}
\end{aligned}
$$

are solved straightforwardly by the first integral

$$
\left(\frac{G}{H}\right)^{2} \mathrm{U}^{\prime 2}+\frac{G}{H}=\text { constant. }
$$

The left-hand side is greater than or equal to $-g_{\text {eff }}^{2} / \mathrm{U}_{0}^{4}$. From Eq. (11), however, a physically meaningful solution results only when the first integral is positive definite. Therefore, we take

$$
\left(\frac{G}{H}\right)^{2} \mathrm{U}^{\prime 2}+\frac{G}{H}=\left(\frac{G}{H}\right)_{*} \equiv \frac{g_{\mathrm{eff}}^{2}}{\mathrm{U}_{0}^{4}} \frac{1}{a^{4}-1},
$$

where the positive first integral is parametrized in terms of $a \equiv \mathrm{U}_{*} / \mathrm{U}_{0} \cdot{ }^{4}$ One may interpret Eq. (25) as a conserved 'energy' of a one-dimensional analog particle for which ' is treated as a time derivative. Simple phase-space consideration shows that the entire configuration of the string lies outside the Schwarzschild horizon, i.e. $U \geqslant U_{0}$.

From the first-integral (25) one obtains an implicit solution

$$
\frac{\mathrm{U}_{0}}{g_{\mathrm{eff}}}\left(x-\frac{d}{2}\right)= \pm \sqrt{a^{4}-1} \int_{a}^{Y} \frac{d y}{\sqrt{\left(y^{4}-1\right)\left(y^{4}-a^{4}\right)}}
$$

where $Y \equiv \mathrm{U} / \mathrm{U}_{0}$ and ranges over $1 \leqslant a \leqslant Y$. Inside the square-root of the integral is a fourth-order polynomial in $y^{2}$. Such an integral can be always brought into a form of an elliptic integral by a change of variable $\omega(y)=\frac{1}{2}\left(y^{2} / a+a / y^{2}\right)$ :

$$
\begin{aligned}
\frac{\mathrm{U}_{0}}{g_{\mathrm{eff}}}\left(x-\frac{d}{2}\right)= & \pm \frac{1}{4 \sqrt{2}} \frac{\sqrt{a^{4}-1}}{\sqrt{a^{3}}} \\
& \times\left[\int_{\gamma}^{z} \frac{d \omega}{\sqrt{(\omega-1)\left(\omega^{2}-\gamma^{2}\right)}}-\int_{\gamma}^{z} \frac{d \omega}{\sqrt{(\omega+1)\left(\omega^{2}-\gamma^{2}\right)}}\right] .
\end{aligned}
$$

Here, $z \equiv \omega(Y)$ and $\gamma \equiv \omega(a)$. Note that $1 \leqslant \gamma \leqslant z$. Explicitly, in terms of elliptic integrals,

\footnotetext{
${ }^{4}$ The zero-temperature limit (which is also the extreme D3-brane limit) is reached by taking $\mathrm{U}_{0} \rightarrow 0$, $a \rightarrow \infty$ while holding $\mathrm{U}_{0}^{4}\left(a^{4}-1\right)$ fixed. Also, positivity of $(G / H)^{2} \mathrm{U}^{2}$ restricts the range of $a$ to $1 \leqslant a \leqslant$ $\mathrm{U} / \mathrm{U}_{0}<\infty$.
} 


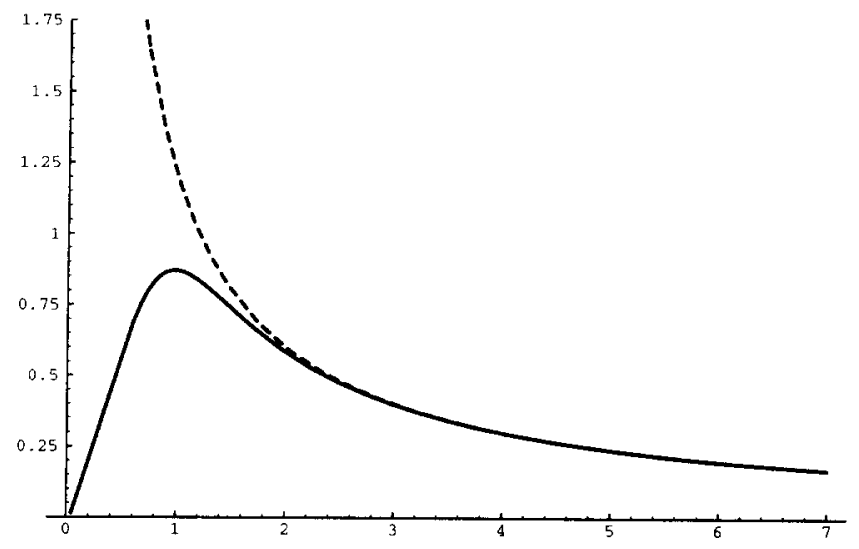

Fig. 2. Functional relation between the inter-quark distance $d$ and $\left(a^{4}-1\right)^{1 / 4}$. For $d \leqslant d_{\max }$, the maximum inter-quark distance, there are two possible U-shape string configurations representing the Wilson-Polyakov loop correlator at two different values of $a$. Also illustrated for comparison is the dashed curve, which represents the same functional relation at zero temperature. Note that, at any $d$, the zero temperature configuration is always unique.

$$
\begin{aligned}
\frac{\mathrm{U}_{0}}{g_{\mathrm{eff}}}\left(x-\frac{d}{2}\right)= & \pm \frac{1}{4 \sqrt{\gamma}} \frac{\sqrt{a^{4}-1}}{\sqrt{a^{3}}} \\
& \times\left[F\left(\sin ^{-1} \sqrt{\frac{z-\gamma}{z-1}}, \sqrt{\frac{\gamma+1}{2 \gamma}}\right)-F\left(\sin ^{-1} \sqrt{\frac{z-\gamma}{z+1}}, \sqrt{\frac{\gamma-1}{2 \gamma}}\right)\right],
\end{aligned}
$$

where $F(\alpha, \beta)$ denotes an elliptic integral of the first kind. As in the zero temperature case, the parameter $a \mathrm{U}_{0} \equiv \mathrm{U}_{*}$ is the U-location for the tip of the U-shaped string. Typical configuration of the string is illustrated in Fig. 1.

The inter-quark distance $d$ is determined by the parameter $a$ :

$$
\frac{\mathrm{U}_{0}}{g_{\mathrm{eff}}} \frac{d}{2}=\frac{1}{4 \sqrt{\gamma}} \frac{\sqrt{a^{4}-1}}{\sqrt{a^{3}}}\left[\mathbf{K}\left(\sqrt{\frac{\gamma+1}{2 \gamma}}\right)-\mathbf{K}\left(\sqrt{\frac{\gamma-1}{2 \gamma}}\right)\right],
$$

where $\mathbf{K}$ denotes the complete elliptic integral of the first kind. The functional relation between $a$ and $d$ is plotted in Fig. 2. The striking difference from zero the temperature limit, which is also plotted in the figure, is the presence of a maximal separation distance, $d \leqslant d_{\max }$, in case the quark pair is connected by the U-shape string. For a fixed $d \leqslant d_{\max }$, Fig. 2 shows that there are two possible $U$-shaped string configurations at two different values of $a$. Recalling that the tip of the U-shape string is located at $a \mathrm{U}_{0}=\mathrm{U}_{*}$ (See Fig. 1), one would expect that a configuration with a larger value of the a parameter has a shorter length, and hence, is an energetically more favorable configuration.

The total energy of the U-shape string of inter-quark separation $d$ is 


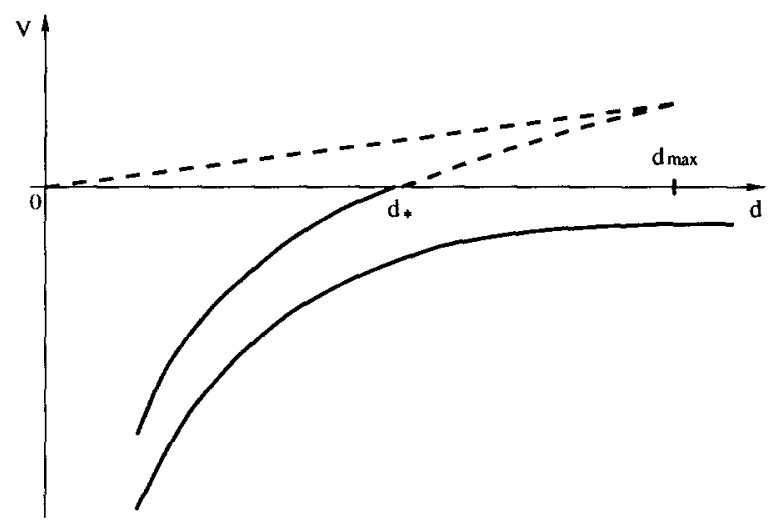

Fig. 3. The heavy quark potential $V_{(\mathrm{Q})}(d)$ defined in Eq. (31) as a function of inter-quark distance $d$. The upper dashed and the lower solid-dashed curves correspond to two distinct string configurations for a given $d \leqslant d_{\max }$ in Fig. 2. The dashed curves are $\mathrm{U}$-shape string configurations that have a higher total energy than the straight parallel string pair. Note that $d_{*}<d_{\max }$. For comparison, the heavy quark potential at zero temperature is shown in the lower solid curve.

$$
E_{(\overline{\mathrm{Q}})}(d, T)=\int_{-d / 2}^{+d / 2} d x \sqrt{\mathrm{U}^{\prime 2}+\frac{H}{G}}=2 \mathrm{U}_{0} \int_{a}^{1} d y \sqrt{\frac{y^{4}-1}{y^{4}-a^{4}}}
$$

which is again regularized by $\Lambda$ introduced in Eq. (14). Among the aforementioned possible string configurations, the lower energy configuration will represent the stable Wilson-Polyakov loop correlator. For the U-shape string configuration, after subtracting the mass of the quark pair, $2 M_{\mathrm{Q}}$, the heavy quark potential is

$$
\begin{aligned}
V_{(\mathrm{Q} \overline{\mathrm{Q}})}(d, T) & \equiv E_{(\mathrm{Q} \overline{\mathrm{Q}})}(d, T)-2 M_{\mathrm{Q}} \\
& =2 \mathrm{U}_{0} \int_{a}^{1} d y \sqrt{\frac{y^{4}-1}{y^{4}-a^{4}}}-2 \mathrm{U}_{0}(\Lambda-1) .
\end{aligned}
$$

The dependence on the inter-quark distance $d$ is obtained by inverting Eq. (29). The result is plotted in Fig. 3 .

In Fig. 3, the energy of the U-shaped string configuration with the smaller $a$-value is plotted as the upper dashed line while the one with the larger $a$-value is shown as the middle solid-dashed curve. The latter is clearly the lower energy configuration. Its potential curve crosses zero at $d=d_{*}$. Once $d \geqslant d_{*}$, Fig. 3 shows that the configuration with two straight strings has a lower energy than the U-shape string configuration. As the two straight strings have no interaction energy, the heavy quark potential vanishes for $d \geqslant d_{*}$. Moreover, for all values of $\mathrm{U}_{0}$, one finds $d_{*}<d_{\max }$. Summarizing, we have found that the two-point correlator of the Wilson-Polyakov loops (5) in gauge theory is described in $a d S_{5}$ supergravity by a $U$-shape string for $d<d_{*}$ and by a pair of straight strings for $d>d_{*}$. 
We now derive an analytical expression for the heavy quark potential. At $d<d_{*}$, $a$ is large: $1 \leqslant a \rightarrow \infty$. Expanding Eq. (29) in powers of $1 / a$, one finds

$$
\frac{\mathrm{U}_{0}}{g_{\text {eff }}} \frac{d}{2}=c\left(\frac{1}{a}-\frac{1}{5 a^{5}}-\frac{1}{10 a^{9}}-\ldots\right),
$$

where

$$
c=\sqrt{2} \mathbf{E}(1 / \sqrt{2})-\frac{1}{\sqrt{2}} \mathbf{K}(1 / \sqrt{2})=\frac{\sqrt{2} \pi^{3 / 2}}{\Gamma(1 / 4)^{2}} \simeq 0.56
$$

and $\mathbf{E}$ denotes the complete elliptic integral of the second kind.

Inverting $\mathrm{Eq}$. (32), one obtains

$$
a(d)=\frac{2 c g_{\mathrm{eff}}}{\mathrm{U}_{0}} \frac{1}{d}-\frac{1}{5}\left(\frac{\mathrm{U}_{0}}{2 c g_{\mathrm{eff}}}\right)^{3} d^{3}+\ldots
$$

Likewise, expanding Eq. (31) in powers of $1 / a$ and using the asymptotic relation Eq. (34), one finally obtains asymptotic expansion of the heavy quark potential:

$V_{(\mathrm{Q} \overline{\mathrm{Q}})}(d, T)= \begin{cases}-4 c^{2} g_{\mathrm{eff}}\left[\frac{1}{d}-\frac{\mathrm{U}_{0}}{2 c^{2} g_{\mathrm{eff}}}+\frac{3}{10}\left(\frac{\mathrm{U}_{0}}{2 c g_{\mathrm{eff}}}\right)^{4} d^{3}+\ldots\right] & \left(d \leqslant d_{*}\right) \\ 0 & \left(d \geqslant d_{*}\right) .\end{cases}$

It is interesting to compare this with the pure $S U(N)$ gauge theory, Eq. (6). Due to thermal excitations, both Eq. (6) and Eq. (35) exhibit short-range interaction. ${ }^{5}$ The two have a different origin, however. The $g_{Y M}$ and $N$ dependence is strikingly different. The $1 /|\mathbf{d}|$ dependence in Eq. (6) is due to non-perturbative magneto-effects, whereas in Eq. (35) it is from the underlying conformal invariance. The conformal invariance also constrains the heavy quark potential $V_{(\mathrm{QQ})} d$ to depend on temperature only through the unique scale-invariant combination ( $T d$ ). Another point to be emphasized is that, at any $d$, the heavy quark potential energy is always higher at finite temperature than at zero temperature (the lowest curve in Fig. 3).

Actually there exists another string configuration that solves equations of motion (23). In fact, the first integral (24) has a separatrix at the Schwarzschild horizon $U=U_{0}$ and the extra solution is a configuration inside the horizon. While its interpretation is not clear, in view of recent claims [21] that large- $N$ gauge theory also covers the Minkowski region behind the Schwarzschild horizon, ${ }^{6}$ the new configuration might be relevant to issues such as resolution of black hole singularities [22]. Hence, we present briefly the configuration and leave its proper interpretation for future work. ${ }^{7}$ Solving Eq. (25) for $0 \leqslant U \leqslant U_{0}$,

\footnotetext{
${ }^{5}$ Recall that, in Eq. (6), the electric mass arises perturbatively but the magnetic mass is a non-perturbative effect.

${ }^{6}$ In fact, Minkowski space-time formulation of finite temperature gauge theory is necessary if one were to study, for example, transport phenomena.

${ }^{7} \mathrm{~J}$. Minahan pointed out that the solution presented in the first version of this paper did not have real energy in Minkowski space-time. The solution actually describes an Euclidean tunnelling. He informed us that he has also found the configuration discussed below.
} 

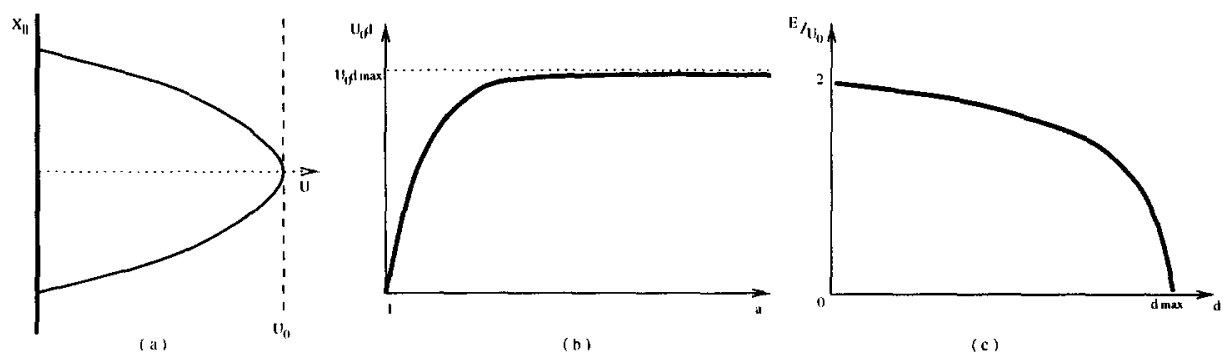

(b)

(c)

Fig. 4. (a) String inside the Schwarzschild horizon; (b) distance $d$ versus the first integral parameter $a$; (c) the total energy as a function of $d$. Note the existence of a maximal distance $d_{\text {max }}$.

$$
\begin{aligned}
\frac{\mathrm{U}_{0}}{g_{\mathrm{eff}}}\left(x-\frac{d}{2}\right)= & \pm \sqrt{a^{4}-1} \int_{a}^{Y} d y \frac{1}{\sqrt{\left(a^{4}-y^{4}\right)\left(1-y^{4}\right)}} ; \quad(0 \leqslant Y \leqslant 1) \\
= & \pm \frac{1}{4 \sqrt{\gamma}} \frac{\sqrt{a^{4}-1}}{\sqrt{a^{3}}} \\
& \times\left[F\left(\sin ^{-1} \frac{\sqrt{2 \gamma}}{\sqrt{z+\gamma}}, \sqrt{\frac{\gamma+1}{2 \gamma}}\right)+F\left(\sin ^{-1} \sqrt{\frac{2 \gamma}{z+\gamma}}, \sqrt{\frac{\gamma-1}{2 \gamma}}\right)\right],
\end{aligned}
$$

where $\gamma=\omega(a)=\frac{1}{2}(a+1 / a)$ and $z \equiv \omega(Y)$. The distance $d$ between two ends of the macroscopic string at $U=0$ is given by

$$
\frac{\mathrm{U}_{0}}{g_{\mathrm{eff}}} \frac{d}{2}=\frac{1}{4 \sqrt{\gamma}} \frac{\sqrt{a^{4}-1}}{\sqrt{a^{3}}}\left[\mathbf{K}\left(\sqrt{\frac{\gamma+1}{2 \gamma}}\right)+\mathbf{K}\left(\sqrt{\frac{\gamma-1}{2 \gamma}}\right)\right] .
$$

As the string extends only inside the horizon, the total energy,

$$
E(a, T)=2 \mathrm{U}_{0} \int_{0}^{1} d y \sqrt{\frac{1-y^{4}}{a^{4}-y^{4}}}
$$

is always finite. In Fig. 4, we have plotted the string configuration, $d$ versus the $a$ parameter, and the energy $E$ as a function of distance $d$.

\section{Near-extremal dynamics of the Born-Infeld quark}

In deducing that the Polyakov-Wilson loop correlator exhibits the expected deconfinement phase behavior at finite temperature, we have taken the interpretation that straight macroscopic strings terminate at the Schwarzschild horizon $\mathrm{U}_{0}$. This is a consequence of Maldacena's observation [18] that near extremal D-branes are located at the horizon, not at the singularity $U=0 .{ }^{8}$ As this phenomenon has played a crucial role in deducing

\footnotetext{
${ }^{8}$ This point has also been stressed in $[20 \mid$.
} 
the behavior of the heavy quark potential, we now would like to examine it from a different angle to gain a better understanding.

Introduce a probe D3-brane in the background of $N$ near extremal D3-branes. Worldvolume dynamics of the probe D3-brane should be sensitive to the position of the $N$ near extremal D3-branes and tell us where they are actually located. To identify that, we consider a single quark state on the probe D3-brane and study its fate as the probe D3-brane passes through the Schwarzschild horizon. Unlike a quark-anti-quark pair, a single quark on a D3-brane carries net color electric flux. At weak coupling $\lambda_{\text {IIB }} \rightarrow 0$, a single quark state is where the Type IIB string is attached and the electric flux is the conserved charge of the fundamental string number [23]. We will now show that the electric flux of an isolated quark becomes unphysical once the probe D3-brane falls inside the Schwarzschild horizon. As this implies that conserved string number becomes unphysical as well, we would conclude that fundamental string cannot penetrate through the horizon.

At strong coupling $g_{\text {eff }} \rightarrow \infty$, isolated quark excitations are accurately described by the large- $N$ resummed quantum Born-Infeld Lagrangian,

$$
L_{\mathrm{DBI}}=-\frac{1}{\lambda_{\mathrm{IIB}}} \int d^{3} x \sqrt{-\operatorname{det}\left(G_{a b}+F_{a b}\right)}-L_{\mathrm{WZ}},
$$

where $G_{a b}=\partial_{a} X^{M} \partial_{b} X^{\nu} G_{\mu \nu}(X)$ denotes the induced metric and $F_{a b}$ is the world-sheet gauge field. The Wess-Zumino term $L_{\mathrm{WZ}}$ provides a volume-dependent self-energy potential. From the Schwarzschild-anti-de Sitter background geometry (2), the quantum Born-Infeld Lagrangian for a probe D3-brane is derived straightforwardly. For spherically symmetric excitations of $\mathrm{U}$ and electric fields only $\left({ }^{\prime} \equiv \partial_{r}, \mathrm{E} \equiv \hat{\mathbf{r}} \cdot \mathbf{E}\right)$ :

$$
L_{\mathrm{DBI}}=-\frac{4 \pi}{\lambda_{\mathrm{IIB}}} \int r^{2} d r \frac{1}{G}\left[\sqrt{H+G \mathrm{U}^{\prime 2}-\frac{G}{H} \dot{\mathrm{U}}^{2}-G \mathrm{E}^{2}}-1\right] .
$$

Canonically conjugate momenta of $U$ and the gauge field are

$$
\begin{aligned}
& \lambda_{\text {IIB }} \Pi_{\mathrm{U}}=\dot{\mathrm{U}} \frac{1 / H}{\sqrt{H+G \mathrm{U}^{2}-\frac{G}{H} \dot{\mathrm{U}}^{2}-G \mathrm{E}^{2}}}, \\
& \lambda_{\text {IIB }} \Pi_{\mathrm{A}}=\mathrm{E} \frac{1}{\sqrt{H+G \mathrm{U}^{\prime 2}-\frac{G}{H} \dot{\mathrm{U}}^{2}-G \mathrm{E}^{2}}} .
\end{aligned}
$$

Thus, restricting to a static configuration, the equations of motion are

$$
\begin{aligned}
\frac{1}{r^{2}}\left(r^{2} \Pi_{\mathrm{A}}\right)^{\prime} & =0, \\
\frac{1}{r^{2}}\left(\frac{r^{2} \mathrm{U}^{\prime}}{\sqrt{H+G \mathrm{U}^{\prime 2}-G \mathrm{E}^{2}}}\right)^{\prime} & =\partial_{\mathrm{U}}\left[\frac{1}{G}\left(\sqrt{H+G \mathrm{U}^{2}-G \mathrm{E}^{2}}-1\right)\right] .
\end{aligned}
$$

The first equation is nothing but the Gauss' law constraint on the world-volume. At zero temperature, $H \rightarrow 1$, and the two equations are solved simultaneously by a first-order BPS equation $E=U^{\prime}[14]$. 
At finite temperature, it is quite difficult to find an exact solution to the equations of motion. Hence, we will consider approximate solutions of an isolated quark, from which useful conclusions can still be drawn. Consider first the limit in which the electric field $\mathrm{E}$ is excited far more than the U-field gradient. From the second Eq. (39), this is a good approximation in so far as $\mathrm{U}$ is not too small. We thus set $\mathrm{U}=\mathrm{U}_{p}$ constant and solve the Gauss' law constraint. The solution is

$$
E(r)=\sqrt{H} \frac{Q_{e}}{\sqrt{r^{4}+r_{0}^{4}}}
$$

where $r_{0}=\left(G Q_{e}^{2}\right)^{1 / 4}$ and $H=H\left(\mathrm{U}_{p}\right), G=G\left(\mathrm{U}_{p}\right)$. The solution Eq. (40) represents an isolated quark of total electric charge $Q_{e}$. The Schwarzschild metric function $H$ plays the role of a dielectric constant and screens the bare electric charge $Q_{e}$. The behavior of $H$, however, depends sensitively on the location $\mathrm{U}_{p}$ of the probe D3-brane. Outside the horizon $\mathrm{U}_{p}>\mathrm{U}_{0}, H>0$ summarizes the effects of thermal excitations. Inside the horizon, $\mathrm{U}_{p}<\mathrm{U}_{0}, H<0$ and the electric field becomes purely imaginary. Moreover, Eq. (37) shows that the energy also becomes purely imaginary. Such pathological behavior is evaded only if D3-branes do not fall inside and stay exactly at the Schwarzschild horizon. Likewise, fundamental strings cannot penetrate through and would end at the horizon too.

The field energy of the isolated quark (40) is calculated from the Lagrangian (36),

$$
\begin{aligned}
E_{\mathrm{Q}} & =\frac{4 \pi}{\lambda_{\mathrm{IIB}}} \int r^{2} d r \frac{1}{G}\left[\sqrt{H-G \mathrm{E}^{2}}-1\right] \\
& =\frac{4 \pi}{\lambda_{\mathrm{IIB}}} \frac{\sqrt{H}}{G} r_{0}^{3} \int_{0}^{\infty} x^{2} d x\left[\sqrt{1-\frac{1}{1+x^{4}}}-\frac{1}{\sqrt{H}}\right] .
\end{aligned}
$$

At the core $x \sim 0$, the integral is completely convergent. As $r_{0}$ depends on the function $G$, one may interpret the ultraviolet finiteness as a consequence of anti-de Sitter background geometry. On the other hand, since $H<1$, the quark energy is infrared divergent at $x \rightarrow \infty$. The infrared divergence is absent at zero temperature, $H=1$. Hence, one may attribute it as a consequence of strong infrared divergence in gauge theory at finite temperature [11]. After regularizing the infrared divergence by replacing the second term in Eq. (41) by unity, one finds that

$$
E_{\mathrm{Q}}^{\mathrm{reg}}:=\frac{3 N}{\lambda_{\mathrm{IIB}}} \frac{\sqrt{H}}{G}\left(\frac{4 \pi}{3} r_{0}^{3}\right), \quad N \approx 0.618 .
$$

The field energy clearly displays the fact that color charge is distributed over a ball of radius $r_{0}$. The field energy on the probe D3-brane vanishes as the brane approaches the Schwarzschild horizon. This is a behavior consistent with Eq. (14) that a pair of straight macroscopic strings ending on the Schwarzschild horizon have no interaction energy.

So far, we have approximated the single quark configuration by suppressing the U-field excitation. While exact considerations are not feasible, it is transparent what modifica- 
tions one might expect for the isolated quark, Eq. (40). Including the contribution of $G \mathrm{U}^{\prime 2}$ to the Lagrangian (37), one recognizes that $\sqrt{H}$ in Eq. (40) is now replaced by an implicit function $\sqrt{H+G^{\prime 2}}$. The new color electric field is physical only if $\mathrm{U}^{\prime 2}+H / G \geqslant 0$. Identifying $r=\sigma$, one finds that this coincides precisely with the first integral (25). Moreover, from Eq. (39), a real-valued U-field configuration is obtained only if $\mathrm{U}^{2}+H / G \geqslant \mathrm{E}^{2}>0$. Again, this results in the condition of Eq. (25). Thus, we conclude that D3-branes and fundamental strings ending on them cannot fall inside the Schwarzschild horizon.

\section{Discussions}

In this paper, we have studied the finite temperature behavior of $d=4, \mathcal{N}=4$ superconformal Yang-Mills theory. Utilizing the correspondence of the large- $N$ and strong coupling limit to Type IIB supergravity compactification on Schwarzschild-antide Sitter space-time, we have studied correlators of the Wilson-Polyakov loop and the heavy quark potential. We have found that the potential exhibits behavior expected for the deconfinement phase at high temperature.

At zero temperature, the timelike Wilson loop is realized by a U-shaped macroscopic string for any interquark separation. At finite temperature, we have seen that the string configuration is either a pair of straight strings or U-shaped. On the supergravity side, the new feature that emerges at finite temperature is the Schwarzschild black hole and its gravitational potential in addition to the zero-temperature, anti-de Sitter gravity. Hence, one may heuristically interpret that the transition from a $\mathrm{U}$-shaped configuration to a pair of straight strings as the interquark distance is increased is a result of pronounced gravitational attraction acting on the macroscopic string.

Crucial to the present study was the fact that macroscopic strings end at the Schwarzschild horizon, not at the singularity. To understand this behavior, we have studied a single quark state on a probe D3-brane in the Schwarzschild-anti-de Sitter space-time. We have found that the state becomes unphysical if the probe D3-brane were to fall into the Schwarzschild horizon. To avoid the pathology, D3-branes as well as fundamental strings ending on them are required to stay on or outside the horizon.

There remain many interesting issues to be explored. Viewing $d=4, \mathcal{N}=4$ superconformal gauge theory as a prototype quenched QCD, it would be most interesting to study in depth issues of confinement and chiral symmetry breaking. We also believe that possible resolutions of the black hole singularity in string theory might be intimately tied to such issues in gauge theory.

\section{Acknowledgements}

We thank G. Horowitz, J. Minahan and S. Yankielowicz for comments on the first version of the paper. S.J.R. and S.T. wish to thank M.R. Douglas, W. Lerche and H. 
Ooguri, organizers of Duality '98 workshop, and the Institute for Theoretical Physics for warm hospitality, where part of this work was accomplished. S.T. also acknowledges useful conversations with S. Förste.

\section{References}

[1] G. 't Hooft, Nucl. Phys. B 72 (1974) 461; Planar Diagram Field Theories, in Progress in Gauge Field Theory, NATO Advanced Study Institute, ed. G. 't Hooft et al. (Plenum, New York, 1984) pp. 271-335.

[2] E. Witten, Nucl. Phys. B 149 (1979) 285; B 160 (1979) 57.

[3| I.R. Klebanov, Nucl. Phys. B 496 (1997) 231;

S.S. Gubser, I.R. Klebanov and A. Tseytlin, Nucl. Phys. B 499 (1997) 217;

S.S. Gubser and I.R. Klebanov, Phys. Lett. B 413 (1997) 41;

M.R. Douglas, J. Polchinski and A. Strominger, Probing five-dimensional black holes with D-branes, hep-th/9703031.

14| J.M. Maldacena, The large $\mathrm{N}$ limit of superconformal field theories and supergravity, hep-th/9711200.

15] S.S. Gubser, I.R. Klebanov and A.M. Polyakov, Gauge theory correlators from non-critical string theory, hep-th/9802109.

16] E. Witten, Anti-De Sitter space and holography, hep-th/9802150.

17] S. Ferrara and C. Fronsdal, Conformal Maxwell theory as a Singleton field theory on $A d S_{5}$, IIB threebranes and duality, hep-th/9712239;

P. Claus, R. Kallosh, J. Kumar, P. Townsend and A. van Proeyen, Conformal theory of M2, D3, M5 and DI +D5-branes, hep-th/9801206;

N. Itzhaki, J. Maldacena, J. Sonnenschein and S. Yankielowicz, Supergravity and the large N limit of theories with 16 supercharges, hep-th/9802042;

S. Ferrara and C. Fronsdal, Gauge fields as composite boundary excitations, hep-th/9802126;

S. Ferrara, C. Fronsdal and A. Zaffaroni, On $N=8$ supergravity on $A d S_{5}$ and $N=4$ superconformal Yang-Mills theory, hep-th/9802203;

G. Horowitz and H. Ooguri, Spectrum of large N gauge theory from supergravity, hep-th/9802116;

S. Kachru and E. Silverstein, 4d conformal field theories and strings on orbifolds, hep-th/9802183;

A. Lawrence, N. Nekrasov and C. Vafa, On conformal field theories in four dimensions, hep-th/9803015;

$\mathrm{O}$. Aharony, $\mathrm{Y}, \mathrm{Oz}$ and $\mathrm{Z}$. Yin, M-Theory on $A d S_{p} \times S^{11-p}$ and superconformal field theories, hepth/9803053;

S. Ferrara and A. Zaffaroni, $N=1,24 \mathrm{D}$ superconformal field theories and supergravity in $A d S_{5}$, hepth/9803060;

M. Bershadsky, Z. Kakushadze and C. Vafa, String expansion as large N expansion of gauge theories, hep-th/9803076.

181 G.T. Horowitz and A. Strominger, Nucl. Phys. B 360 (1991) 197.

19| A.M. Polyakov, Phys. Lett. B 72 (1978) 477.

[10] L. Susskind, Phys. Rev. D 20 (1979) 2610.

111 D.J. Gross, R.D. Pisarski and L.G. Yaffe, Rev. Mod. Phys. 53 (1981) 43.

[12] B. Svetitsky, Phys. Rep. 132 (1986) 1.

[13] E. Braaten and A. Nieto, Phys. Rev. Lett. 74 (1995) 3530.

[14] S.-J. Rey and J.-T. Yee, Macroscopic strings as heavy quarks in large N gauge theories and anti-de Sitter supergravity, hep-th/9803001.

[15] J. Maldacena, Wilson loops in large N field theories, hep-th/9803002.

[16] J.A. Minahan, Quark-monopole potentials in large $N$ Yang-Mills, hep-th/9803111.

[17] O. Aharony, J. Sonnenschein and S. Yankielowicz, Nucl. Phys. B 474 (1996) 309;

J.H. Schwarz, Nucl. Phys. [Proc. Suppl.] B 55 (1997) 1;

K. Dasgupta and S. Mukhi, BPS nature of 3-string junctions, hep-th/9711094;

A. Sen, String Network, hep-th/9711130;

S.-J. Rey and J.-T. Yee, BPS dynamics of triple $(p, q)$ string junction, hep-th/9711202;

M. Krogh and S. Lee, String network from M-theory, hep-th/9712050;

Y. Matsuo and K. Okuyama, BPS condition of string junction from M theory, hep-th/97/2070; 
O. Bergman, Three-pronged strings and 1/4 BPS states in $N=4$ Super-Yang-Mills, hep-th/9712211; M.R. Gaberdiel, T. Hauer and B. Zwiebach, Open string-string junction transitions, hep-th/9801205;

C.G. Callan Jr. and L. Thorlacius, World-sheet dynamics of string junctions, hep-th/9803097.

[18] J. Maldacena, Phys. Rev. D 57 (1998) 3736.

$119 \mid$ E. Witten, Anti-de Sitter space, thermal phase transition and confinement in gauge theories, hepth/9803131.

$[20]$ A. Brandhuber, N. Itzhaki, J. Sonnenschein and S. Yankielowicz, Wilson loops in the large N limit at finite temperature, hep-th/9803137.

121) G.T. Horowitz and H. Ooguri, Spectrum of large N gauge theory from supergravity, hep-th/9802116.

122| G.T. Horowitz and S.F. Ross, Possible resolution of black hole singularities from large- $N$ gauge theory, hep-th/9803085.

[23| E. Witten, Nucl. Phys. B 460 (1996) 335. 